\title{
Self-shaping of oil droplets via the formation of intermediate rotator phases upon cooling
}

\author{
Nikolai Denkov ${ }^{1}$, Slavka Tcholakova ${ }^{1}$, Ivan Lesov ${ }^{1}$, Diana Cholakova ${ }^{1} \&$ Stoyan K. Smoukov ${ }^{2}$ \\ ${ }^{1}$ Department of Chemical and Pharmaceutical Engineering, Faculty of Chemistry and Pharmacy, Sofia \\ University, 1164 Sofia, Bulgaria.
${ }^{2}$ Active and Intelligent Materials Laboratory, Department of Materials Science \& Metallurgy, University of Cambridge, Cambridge, CB3 OFS, UK.

Revealing the chemical and physical mechanisms underlying symmetry breaking and shape transformations is key to understanding morphogenesis ${ }^{1}$. If we are to synthesize artificial structures with similar control and complexity to biological systems, we need energy- and material-efficient bottom-up processes to create building blocks of various shapes that can further assemble into hierarchical structures. Lithographic top-down processing ${ }^{2}$ allows a high level of structural control in microparticle production but at the expense of limited productivity. Conversely, bottom-up particle syntheses ${ }^{3-8}$ have higher material and energy efficiency, but are more limited in the shapes achievable. Linear hydrocarbons are known to pass through a series of metastable plastic rotator phases before freezing ${ }^{9,10}$. Here we show that by using appropriate cooling protocols, we can harness these phase transitions to control the deformation of liquid hydrocarbon droplets and then freeze them into solid particles, permanently preserving their shape. Upon cooling, the droplets spontaneously break their shape symmetry several times, morphing through a series of complex regular shapes owing to the internal phasetransition processes. In this way we produce particles including micrometre-sized octahedra, various polygonal platelets, $O$-shapes, and fibres of submicrometre diameter, which can be selectively frozen into the corresponding solid particles. This mechanism offers insights into achieving complex morphogenesis from a system with a minimal number of molecular components.

We illustrate the capabilities of this new approach by using droplets of different linear hydrocarbons with 14-20 carbon atoms (namely from tetradecane to eicosane). The alkanes were pre-dispersed as droplets in $1.5 \mathrm{wt} \%$ aqueous surfactant solution, which can afterwards be transformed into a variety of solid particles with different shapes through the choice of appropriate surfactants and controlled cooling rates (Fig. 1 and Extended Data Fig. 1). Figure 2 shows how the choice of surfactant can influence the particle shape and aspect ratio. Upon 
freezing, many of the obtained thin, high-aspect-ratio structures develop a puncture hole in their interior, due to the volumetric shrinkage accompanying solidification.

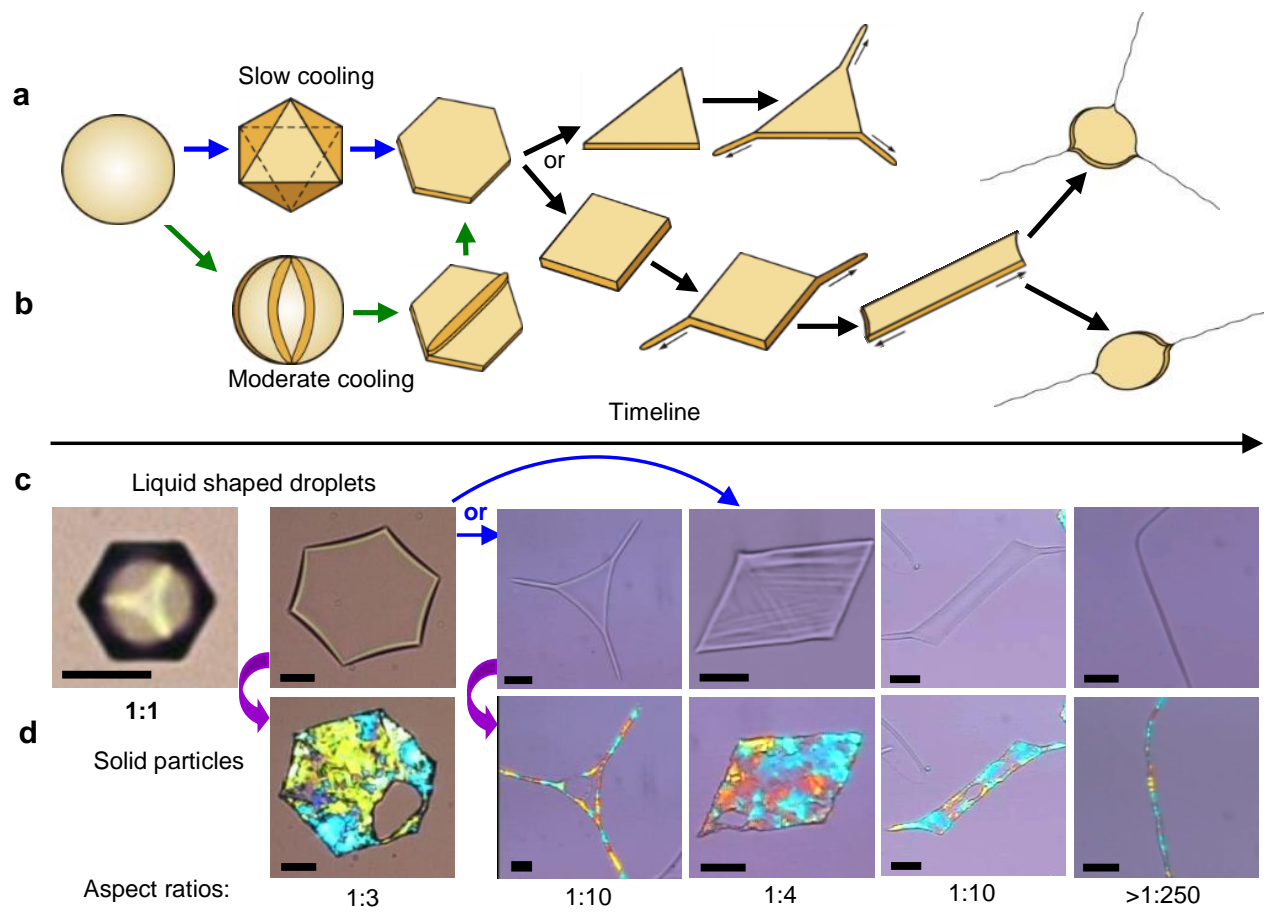

Figure 1 Schematic of the shape transformations observed during cooling of emulsion droplets of pure hydrocarbons in water, in the presence of $1.5 \mathrm{wt} \%$ surfactant. a, Under slow cooling the spheres transform consecutively into regular octahedra, hexagonal platelets, triangular (or tetragonal) platelets, platelets with long asperities and, eventually, thin fibres. $\mathbf{b}$, Under moderate cooling the droplet surface corrugates and forms a hexagonal platelet with protrusions, before forming the regular hexagonal platelet. c, Hexadecane liquid droplets in Brij 58 solution at different stages. d, Solid particles with different shapes, obtained at appropriate cooling rates. Scale bars, $20 \mu \mathrm{m}$.

Our experiments show that the drop-shape transformations and the final shape of the frozen particles depend most importantly on three factors: surfactant type, cooling rate, and initial droplet size. We outline the main effects of these control factors and, afterwards, we explain the basic mechanism of the drop shape evolution.

Surfactants are amphiphilic molecules with a hydrophilic head-group (ionic or nonionic) and hydrophobic alkyl chain. While only two of our surfactants were ultra-pure (CTAB and $\mathrm{C}_{14} \mathrm{H}_{29} \mathrm{SO}_{4} \mathrm{Na}$ with purity $>99 \%$ ), our experiments with more than ten surfactants of all types (anionic, cationic and non-ionic) showed the same general sequence of shape transformations (Fig. 1). These transformations occurred only when the surfactant chain length was similar to or longer than the length of the hydrocarbon molecules in the droplets. Such long-chain surfactants can freeze in the adsorption layer on the drop-water interface, 
before the freezing of the alkane in the droplet interior ${ }^{10}$, and thus have a critical role in the formation of drops with non-spherical shapes. The use of surfactants with shorter chain lengths led to drops freezing into spherical solid particles, without any peculiar shape transformations.

The rate of cooling is another crucial factor in the observed phenomenon. Upon slow and moderate cooling rates (below about $4 \mathrm{~K} \mathrm{~min}^{-1}$ ), the spherical hydrocarbon drops undergo a series of shape transformations. Figure 1 illustrates the case of hexadecane drops in water containing $1.5 \mathrm{wt} \%$ of the non-ionic surfactant Brij 58. Initially, the spherical drops transform into regular octahedra (regular polyhedra whose surface is shaped by eight triangular facets) that then transform into flat platelets with a hexagonal base. Upon further cooling, these hexagons transform either into triangular or tetragonal platelets, the ratio of which depends on the surfactant, the cooling rate, and the initial size of the droplets. Subsequently, rod-like asperities with diameters of around $5 \mu \mathrm{m}$ appear and grow into long filaments from the platelet tips. Finally, if the cooling is sufficiently slow (less than $0.5 \mathrm{~K} \mathrm{~min}^{-1}$ ), these asperities elongate further to form very thin fibres with diameters of around $0.5 \mu \mathrm{m}$. When the cooling rates were varied between 0.01 and $2 \mathrm{~K} \mathrm{~min}^{-1}$, each transformation took between $30 \mathrm{~s}$ and several minutes (see Supplementary Video 1). Depending on the cooling rate, the drops freezing into solid particles occurred at different stages of this evolution path-slower cooling led to freezing at a later stage. Thus, using an appropriate intermediate cooling rate, we could transform an intermediate drop shape into a solid frozen particle with the same shape (Figs 1 and 2; alternatively, one can apply step-acceleration cooling to freeze the deformed drops). For example, using Brij 58 as surfactant, at $0.2 \mathrm{~K} \mathrm{~min}^{-1}$ cooling with $30 \mu \mathrm{m}$ droplets, we obtained $25 \pm 5 \%$ triangles and $75 \pm 5 \%$ rhomboids that evolve into rod-shape particles and then finally into fibres, as determined from observations of over 100 droplets in more than 10 independent experiments. For comparison, when using $10 \mu \mathrm{m}$ droplets with Tween 60, at $0.2 \mathrm{~K} \mathrm{~min}^{-1}$ cooling, we could yield more than $90 \%$ rod-shape particles.

Drop size was another important factor. The images shown in Figs 1 and 2 are obtained with drops of initial diameter around $20 \mu \mathrm{m}$. Very similar results were obtained with drops of diameter between 1 and $50 \mu \mathrm{m}$. At low cooling rates $\left(0.01-2 \mathrm{~K} \mathrm{~min}^{-1}\right)$, both small and large drops evolved in shape when appropriate surfactants were used. However, at higher cooling rates the big drops tended to freeze into spherical solid particles without shape transformations, while the smaller drops readily evolved in shape. Thus we could induce shape transformations of drops with diameter $1-50 \mu \mathrm{m}$ in a wide range of cooling rates $(0.01-$ 
$2 \mathrm{~K} \mathrm{~min}^{-1}$ ). Note that the small droplets with micrometre size are involved in intensive Brownian motion that, however, does not suppress the shape transformations. The investigation of submicrometre droplets was postponed for a separate study because the observation of their evolution requires much more sophisticated experimental methods.

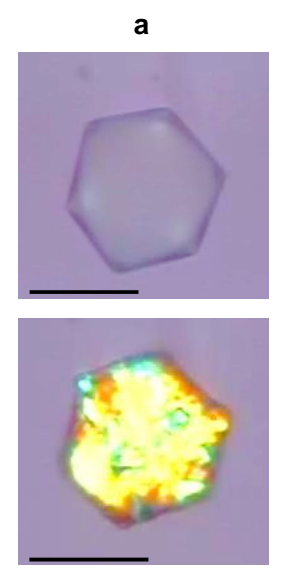

$1: 2$ b
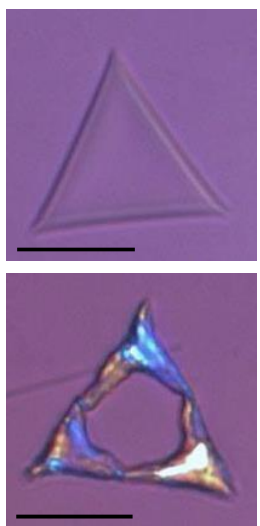

$1: 3$ c

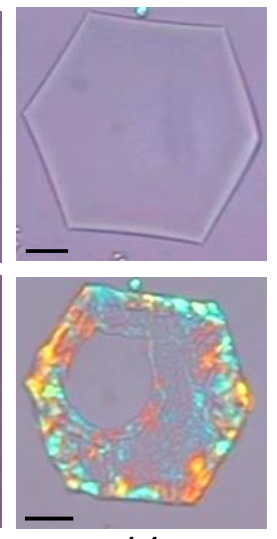

$1: 4$ d

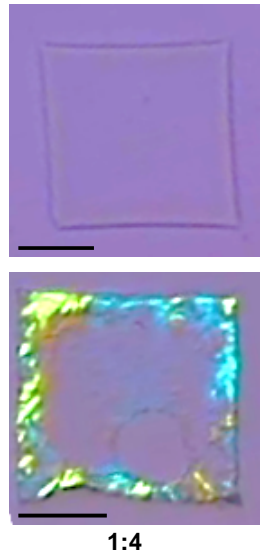

e

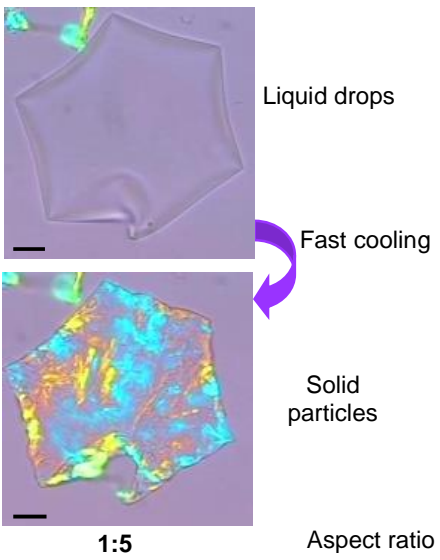

Figure 2 Choice of surfactant and cooling rate can determine particle shape and aspect ratio. a-e, Microscope images of deformed liquid droplets (top) and of the solid particles obtained from these droplets (bottom), upon cooling of hexadecane-in-water emulsions, stabilized by various surfactants: a, b, Tween 60; c, d, Tween 40; e, Brij 78. The numbers indicate the aspect ratio. The aspect ratio of the various shapes along the drop evolution (compare with Fig. 1) depends on the specific surfactant used to stabilize the drops. Scale bars, $20 \mu \mathrm{m}$.

The observed numerous shape transformations are surprisingly governed by a single mechanism. It was deduced from the experimental observations described earlier as follows.

The requirement for a long surfactant-chain length indicates that the shape transformations are triggered by the freezing surfactant adsorption layers, formed on the hydrocarbon-water interface. Numerical estimates described later demonstrate that these adsorption layers do not possess a sufficiently high bending moment to deform the hydrocarbon drops. Therefore, before freezing, the formation of mesomorphic hydrocarbon phases, just inside the surface of the liquid drops, is required to trigger the observed transformations. Indeed, the drop-shape transformations start around the freezing temperature of the bulk hydrocarbon phase transition $\left(18^{\circ} \mathrm{C}\right.$ for hexadecane). In this temperature range, linear hydrocarbons form so-called 'rotator' mesomorphic phases, which represent a class of 
plastic phases in which the molecules possess long-range translational order, yet rotate freely around their long axis ${ }^{9,10}$. Owing to their positional long-range order, the rotator phases generate non-isotropic elastic stresses, which are sufficiently high to deform liquid drops, overcoming their interfacial tension ${ }^{11}$.

Images of deformed drops clearly illustrate the elastic nature of the liquid material confined inside the drops (Fig. 3 and Supplementary Video 2). For non-elastic materials, the long filaments growing from the tips of the triangular platelet shown in Fig. 3a would be unstable and should break into small spherical droplets through so-called 'Plateau-Rayleigh' capillary instability, under the action of capillary pressure, which destabilizes cylindrical liquid jets ${ }^{12}$. The angular doughnut-shaped droplets, shown in Fig. 3b, would also be unstable in shape, unless the drop material possesses elasticity to counteract the capillary pressure, forcing the common liquid drops to acquire a spherical shape.
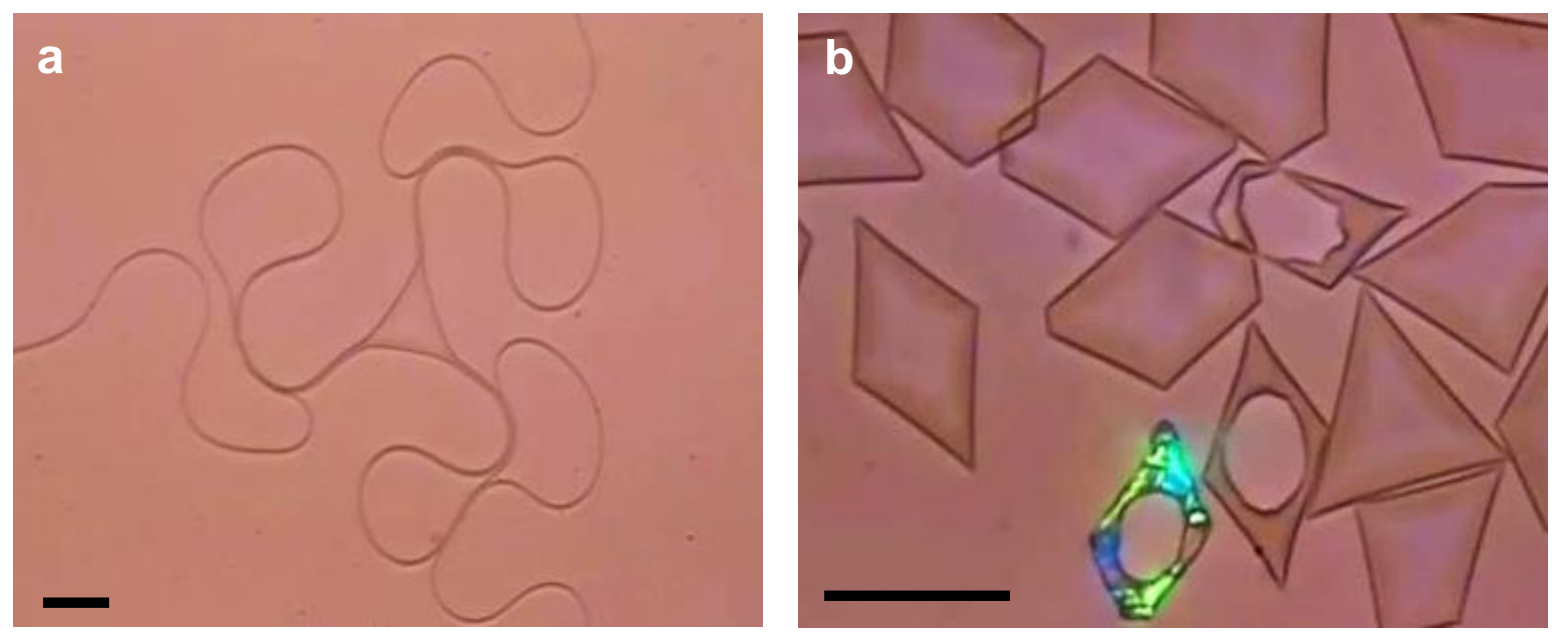

Figure 3 Illustrative examples of liquid drop shapes that would be unstable in the absence of elastic properties of the fluid drop material. a, Image of a triangular platelet with very long cylindrical asperities, protruding from the platelet tips. b, Image of rhomboidshaped liquid drops, some with a hole in their centre, a shape clearly not governed by surface tension only. Such images prove that the fluiddrops contain plastic phases, while still containing liquid inside (the oval shape in the puncture determined by surface tension). The coloured, bottom-most shape is the only one frozen. Scale bars, $50 \mu \mathrm{m}$.

The simplest possible explanation of the observed non-spherical drops could be that the rotator phases, structured inside the entire volume of the drop interior, create high elastic stresses that deform the drop surface. However, some of our results contradict this simplest explanation. For example, the optical microscopy observations of the liquid droplets of various shapes under cross-polarized light showed only faint colours, in contrast to the 
characteristic intense colours that are typically observed for thick non-isotropic liquid crystal layers $^{13}$. If the interior of the deformed liquid drops were entirely filled with an anisotropic plastic phase, the liquid drops would have appeared more coloured in cross-polarized light. We do observe the appearance of beautiful intense colours, but only in the moment of complete freezing of the droplets, indicating the formation of crystal domains in the frozen particles (Figs 1 and 2).

From these results we concluded that the freezing surfactant adsorption layer induces the formation of a thin layer of a hydrocarbon plastic rotator phase of thickness $h_{\mathrm{PL}}$, adjacent to the drop surface, which in turn, drives the observed drop-shape transformations (Fig. 4). Surface-induced formation of liquid crystal phase layers has been observed previously ${ }^{14,15}$ and, as shown later, such plastic interfacial sheets possess a sufficiently large bending moment, able to counteract the effects of the hydrocarbon-water interfacial tension that enforces the spherical shape of the common liquid drops. Being submicrometre in thickness, these sheets appear only with faint colours in cross-polarized light ${ }^{16}$, just as observed in our experiments.

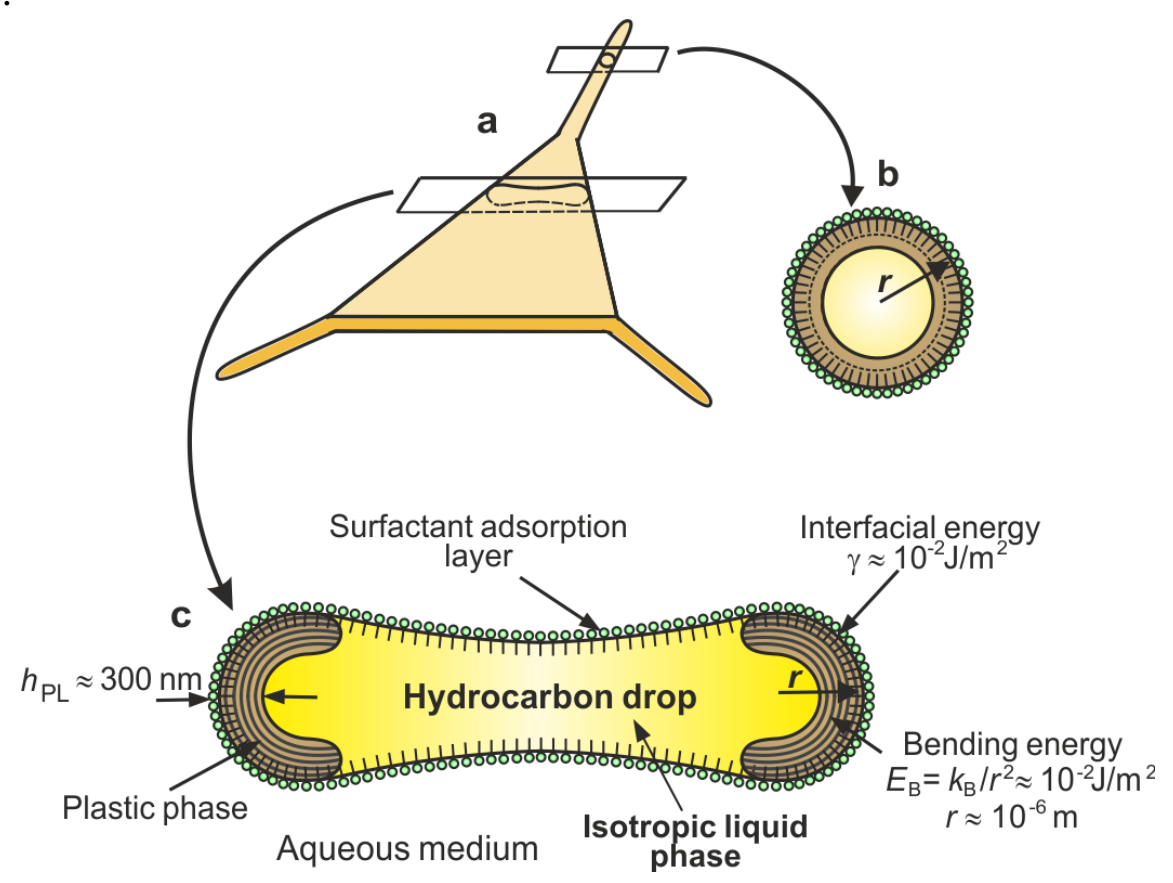

Figure 4 Schematic presentation of the drop-shape deformation mechanism, driven by the formation of interfacial plastic phases. a-c, Cross-sections of a platelet-shaped particle with protrusions (a) are shown in $(\mathbf{b}, \mathbf{c})$. In the presence of appropriate surfactant, thin plastic phases (brown regions) with thickness $h_{\mathrm{PL}}$, bending elasticity constant $K_{\mathrm{B}}$ and characteristic curvature of the shaped droplet edges, $r$, form at the hydrocarbon-water interface, adjacent to the surfaces with high curvature. The low-temperature-induced, highly curved plastic phases form an energetically favourable expanding frame at the drop edges, which drives the observed shape transformations. For clarity, the figure is not to scale. 
The thickness of these plastic interfacial sheets, $h_{\mathrm{PL}}$, could be estimated by considering the balance of the bending and surface area energies of the drop surface. The surface area energy is represented by the interfacial tension, $\gamma$, which was measured to be between 5 and $10 \mathrm{~mJ} \mathrm{~m}^{-2}$ for the surfactant-containing systems studied. The bending energy per unit area of the hydrocarbon-water interface could be estimated ${ }^{17,18}$ as $E_{\mathrm{B}} \approx K_{\mathrm{B}} / r^{2}$, where $K_{\mathrm{B}}$ is the bending elasticity constant and $r \approx 1 \mu \mathrm{m}$ is the observed characteristic curvature of the shaped droplet edges. Such stable, highly deformed droplets could be formed only if the highly curved phases are energetically favourable, and if $E_{\mathrm{B}}$ is comparable or bigger than the surface tension $\gamma$ (both measured in $\mathrm{J} \mathrm{m}^{-2}$ ), which would pull the surface back to the lower spherical curvature. This leads to the minimum estimate for $K_{\mathrm{B}} \approx 10^{-14} \mathrm{~J}$, a value much higher than the known ${ }^{17,18}$ bending constants of frozen lipid bilayers or surfactant adsorption monolayers, $K_{\mathrm{B}} \approx 10^{-18} \mathrm{~J}$. Taking into account ${ }^{18}$ that $K_{\mathrm{B}}$ is proportional to $h_{\mathrm{PL}}^{2}$, we estimate that $h_{\mathrm{PL}} \approx 300 \mathrm{~nm}$ for the observed deformed drops. Similar values of $h_{\mathrm{PL}}$ were reported in independent experimental studies ${ }^{14,15}$ for surface-induced liquid crystal sheet phases. As already explained, the bending forces resulting from $h \approx 1.5-3 \mathrm{~nm}$ for surfactant monolayers and lipid bilayers ${ }^{17,18}$, and the respective bending moment of $K_{\mathrm{B}} \approx 10^{-18} \mathrm{~J}$, are far too weak to deform liquid drops for the interfacial tension values measured in our systems.

The elastic layers seem mostly localized at the shape edges and are characterized also by their 'spontaneous curvature' ${ }^{17,19}$, that is, the curvature that the interface would acquire if no other forces (besides the local intermolecular forces) were involved. All our observations show that the shape transformations are driven by the growth of edges with high spontaneous curvature (small radius of curvature) in one direction, thus forming cylindrical structures in the drop edges. These energetically favourable, cylindrical plastic crystal phases grow in length, thus forming elastic frames that overcome the interfacial tension and stretch the droplets to flatter shapes with high aspect ratios. Indeed, as illustrated in Fig. 3 and Supplementary Video 2, upon puncture, the inside of the rhomboid liquid droplets acquire the minimum circumference dictated by interfacial tension, while preserving intact the outside shaped frame. Figure 1 and Supplementary Video 1 show how the plastic crystals grow and disproportionate into different straight edges to form droplet shapes with longer and longer circumferences, until finally forming highly elongated drops and thin fibres of radius less than $0.4 \mu \mathrm{m}$. Such fibres contain all the material in a single 'edge' region, probably all composed of a rotator phase and providing an estimate for the spontaneous curvature of these phases. 
Our observations with droplets of several linear hydrocarbons show that no specially designed molecules are needed to observe this drop 'self-shaping' phenomenon. By selecting hydrocarbons with appropriate chain length, we varied the temperature range for the liquid drop transformations: the tetradecane drops had non-spherical shapes in a range of 0 to $3{ }^{\circ} \mathrm{C}$, hexadecane drops in a range of 9 and $18^{\circ} \mathrm{C}$, and eicosane drops in a range of 30 to $35^{\circ} \mathrm{C}$.

The growth of smectic liquid crystalline fibres in CTAB solutions above the critical micellar concentration has previously been observed ${ }^{20,21}$. However, in these papers only one type of structure was observed (rod-shaped particles) whereas we are able to produce particles with a wide variety of shapes in a controlled manner. The drop-shape sequence of transformations we observe is much richer and probably different in mechanism from those observed previously ${ }^{20,21}$. Also, hexagonal drop shapes of lyotropic liquid crystal phases have been observed before ${ }^{11}$. However, the full array of complex shapes shown in Figs 1 and 2, and the possibility of transforming between them and of capturing them in a frozen state, are novel (see also Extended Data Figs 1 and 2).

Our approach can be used to produce 'shape-on-demand' particles, noting that highaspect-ratio micro/nanoparticles show preferential internalization in tumour cells ${ }^{22}$ and that tissue/organ uptake can be shape specific ${ }^{23}$. A combination with microfluidic techniques ${ }^{6,24,25}$ seems particularly suitable to explore the full range of such opportunities and the self-shaping method's governing mechanism of symmetry breaking. By controlling the local temperature profile in the microfluidic channel, custom shaped particle populations or mixtures of specific shapes and various sizes could be produced. The obtained shaped particles and fibres could be used to build hierarchical structures or as sacrificial templates for the production of porous materials with complex morphologies.

We report a novel bottom-up mechanism for morphogenesis and an energy- and material-efficient method for the formation of micro- and nanoscale liquid drops and solid particles with complex shapes. The ability of a single fluid phase to form spontaneously the wide variety of shapes we report could decrease the perceived informational complexity of many structures ${ }^{26}$. This shape-shifting is probably used in nature, it is of clear relevance to the emerging field of active matter, and is expected to be applicable to other rotator-phase- and plastic-phase-forming molecules and biomolecules.

The morphogenesis mechanism we present is expected to stimulate research in a number of fields, as the observed phenomena combine several active research areas, such as capillarity and elasticity, liquid crystal and plastic phases in confined spaces, and surface and 
bulk nucleation. The process is probably a good platform for investigating phase equilibria, the role of confinement and the melting of two-dimensional crystals, as well as the interplay between liquid crystal defects and surface bending elasticity resulting in shape changes ${ }^{27,28}$. It is of particular interest for elucidating novel mechanisms of symmetry breaking that contribute to understanding the fundamental processes of morphogenesis.

Received 11 June; accepted 27 October 2015.

Published online 9 December 2015.

Published 17 December 2015.

1. Turing, A. M. The Chemical Basis of Morphogenesis. Philos. Trans. R. Soc. London 237, 37-72 (1952).

2. Champion, J. A., Katare, Y. K. \& Mitragotri, S. Particle shape: a new design parameter for micro- and nanoscale drug delivery carriers. J. Control. Release 121, 39 (2007).

3. Xiao, J. \& Qi, L. Surfactant-assisted, shape-controlled synthesis of gold nanocrystals. Nanoscale 3, 1383-1396 (2011).

4. Peng, X. et al. Shape control of CdSe nanocrystals. Nature. 2, 145-150 (2003).

5. Alargova, R.G., Bhatt, K.H., Paunov \& V. N., Velev, O.D. Scalable synthesis of a new class of polymer microrods by a liquid-liquid dispersion technique. Adv. Mater. 16, 1653-1657 (2004).

6. Dendukuri, D. \& Doyle, P. S. The synthesis and assembly of polymeric microparticles using microfluidics. Adv. Mater. 21, 4071-4086 (2009).

7. Deitzel, J. M., Kleinmeyer, J., Harris, D. \& Beck Tan, N.C. The effect of processing variables on the morphology of electrospun nanofibers and textiles. Polymer 42, 261272 (2001).

8. Smoukov, S. K. et al. Scalable Liquid Shear-Driven Synthesis of Polymer Nanomaterials, Adv. Mater. 27, 2642-2647 (2015).

9. Sirota, E. B. \& Herhold, A. B. Transient phase-induced nucleation. Science 283, 529532 (1999).

10. Ueno, S., Hamada, Y. \& Sato, K. Controlling Polymorphic Crystallization of n-Alkane Crystals in Emulsion Droplets through Interfacial Heterogeneous Nucleation. Cryst. Growth Des. 3, 935-939 (2003).

11. Jeong, J., Davidson, Z. S., Collings, P. J., Lubensky, T. C. \& Yodh, A.G. Chiral symmetry breaking and surface faceting in chromonic liquid crystal droplets with giant elastic anisotropy. Proc. Natl. Acad. Sci. USA 111, 1742-1747 (2014).

12. Stone, H. Dynamics of drop deformation and breakup in viscous fluids. Annu. Rev. Fluid Mech. 26, 65-102 (1994).

13. Zhang, L. Y., Zhang, Q. K. \& Zhang, Y. D. Design, synthesis, and characterisation of symmetrical bent-core liquid crystalline dimers with diacetylene spacer. Liq. Cryst. 40, 1263-1273 (2013).

14. Wittebrood, M. M., Luijendijk, D. H., Stallinga, S., Rasing, Th. \& Musevic, I. Thickness-dependent phase transition in thin nematic films. Phys. Rev. E Stat. Phys. Plasmas Fluids Relat. Interdiscip. Topics 54, $5232-5234$ (1996). 
15. Miyano, K. Surface-induced ordering of a liquid crystal in the isotropic phase. $J$. Chem. Phys. 71, 4108-4111 (1979).

16. Bloss, F. D. An Introduction to the Methods of Optical Crystallography (Holt, Rinehart and Winston, 1961).

17. Israelachvili, J. N. Intermolecular and Surface Forces (Academic, 2011).

18. Evans, E. A. \& Skalak, R. Mechanics and Thermodynamics of Biomembranes. (CRC, 1980).

19. Jung, H. T., Coldren, B., Zasadzinski, J. A., Iampietro, D. J. \& Kaler, E. W. The origins of stability of spontaneous vesicles. Proc. Natl. Acad. Sci. USA 98, 1353-1357 (2001).

20. Peddireddy, K. et al. Lasing and waveguiding in smectic A liquid crystal optical fibers. Opt. Express 21, 30233-30242 (2013).

21. Peddireddy, K., Kumar, P., Thutupalli, S., Herminghaus, S. \& Bahr, C. Myelin structures formed by thermotropic smectic liquid crystals. Langmuir 29, 15682-15688 (2013).

22. Gratton, S. E. et al. The effect of particle design on cellular internalization pathways. Proc. Natl Acad. Sci. USA 105, 11613-11618 (2008).

23. Decuzzi, P. et al. Size and shape affects in the biodistribution of intravascularly injected particles. J. Control. Release 141, 320-327 (2010).

24. Makgwane, P. R., Ray, S. S. Synthesis of nanomaterials by continuous-flow microfluidics: a review. J. Nanosci. Nanotechnol. 14, 1338-1363 (2014).

25. Kim, J.-W., Utada, A. S., Fernández-Nieves, A., Hu, Z. \& Weitz, D. A. Fabrication of monodisperse gel shells and functional microgels in microfluidic devices. Angew. Chem. Int. Ed. 46, 1819-1922 (2007).

26. Neville, A. C. Molecular and mechanical aspects of helicoid development in plant cell walls. BioEssays 3, 4-8 (1985).

27. Li, Y., Miao, H., Ma, H. \& Chen, J. Z. Y. Topological defects of tetratic liquid-crystal order on a soft spherical surface Soft Matter 9, 11461-11466 (2013).

28. Bowick, M. J., Giomi, L. Two-dimensional matter: order, curvature and defects, $A d v$. Phys. 58, 449-563 (2009).

Acknowledgements This work was funded by the European Research Council (ERC) grant to S.K.S., EMATTER (\#280078). The study falls under the umbrella of European network COST MP 1106 and the capacity building project BeyondEverest of the European Commission (grant no. 286205).

Author Contributions N.D. and S.T. conceived the main idea for the study, designed the experiments and performed most of the result interpretation, N.D. wrote the first draft, I.L. and D.C. performed the experiments and prepared the figures, S.K.S. contributed important ideas for the experiments and data and mechanism interpretation, and edited the text.

Author Information Reprints and permissions information is available at www.nature.com/reprints. The authors declare no competing financial interests. Readers are welcome to comment on the online version of the paper. Correspondence and requests for materials should be addressed to S.K.S. (sks46@cam.ac.uk). 


\section{METHODS}

The alkanes (>99\% purity) were purchased from Sigma-Aldrich, and further purified by passing several times through columns of Florisil to remove the polar components. In the absence of surfactant, hydrocarbon-water interfacial tension was measured to be always above $50 \mathrm{mN} \mathrm{m}^{-1}$ as known for pure alkanes. The hydrocarbon-water interfacial tension (water containing $1.5 \mathrm{wt} \%$ surfactant), $\gamma$, was measured by drop-shape analysis (instrument DSA100 by Krüss) to be in the range between 5 and $10 \mathrm{mN} \mathrm{m}^{-1}$ for all surfactant systems studied, in the entire range from room temperature down to the temperatures of drop deformation and freezing.

The original hydrocarbon-in-water emulsions were prepared by membrane emulsification with 2, 3, 5 or $10 \mu \mathrm{m}$ pore-size glass membrane (SPG) in $1.5 \mathrm{wt} \%$ solutions of Brij 58 (Fig. 1), Tween 40, Tween 60 or Brij 78 (Fig. 2 and Extended Data Fig. 1), CTAB (Extended Data Fig. 1) or other surfactants (images not shown). All surfactants were chosen to be water soluble with high hydrophilic-lipophilic balance (HLB >14), so the surfactant would be almost exclusively in the water phase. Extended Data Table 1 summarizes the HLB values.

The emulsion cooling was realized in rectangular glass capillaries with length of $50 \mathrm{~mm}$, width of $1 \mathrm{~mm}$ and height of $0.1 \mathrm{~mm}$, enclosed within a custom-made metal cooling chamber, with optical windows for microscope observation (Extended Data Fig. 4). The chamber temperature was controlled by cryo-thermostat (Julabo CF30) and measured close to the emulsion location, using a calibrated thermo-couple probe with an accuracy of $\pm 0.2{ }^{\circ} \mathrm{C}$.

The optical observations were performed with Axioplan and AxioImager M2.m microscopes (Zeiss) in transmitted, cross-polarized white light, with included compensator plate situated after the sample and before the analyser, at $45^{\circ}$ with respect to both the analyser and the polarizer. Long-focus objectives $\times 20$ and $\times 50$ were used. The drop diameter was determined from microscope images.

The average 'height' of the deformed drops was calculated by dividing the total volume of the drop (calculated from the radius of the initial spherical drops) by the projected area of the non-spherical drop shapes, measured from the microscope images. In this way the aspect ratios, shown in Figs 1 and 2, were determined. 


\section{Supplementary Videos}

Supplementary Video SV1. Video with the shape transformations of hexadecane-in-water drops, stabilized by the nonionic surfactant Brij 58 and cooled with rate of $0.4 \mathrm{~K} / \mathrm{min}$. This video illustrates the drop-shape evolution shown in Figures 1 and 2.

Supplementary Video SV2. Video with the shape transformation of hexadecane-in-water drops, stabilized by the nonionic surfactant Tween 60 and cooled with rate of $0.4 \mathrm{~K} / \mathrm{min}$. This video demonstrates the formation of doughnut-shaped drops which could exist only if the drops contain liquid-crystalline material with certain elastic properties.

\section{Extended Data Figures}
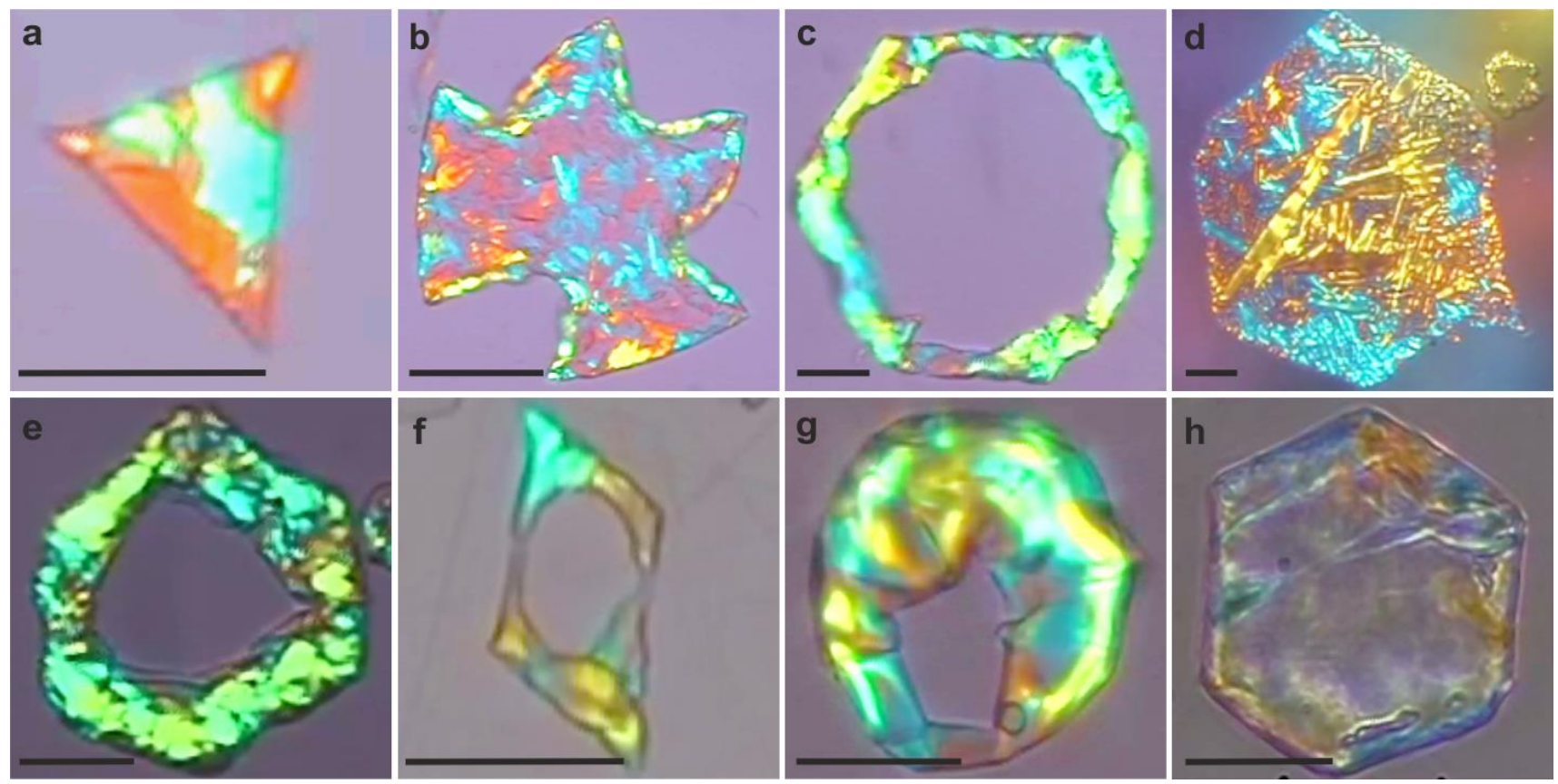

Extended Data Figure 1 Solid particles with various shapes. $\mathbf{a}-\mathbf{h}$, The shapes were obtained by freezing of deformed hexadecane $(\mathbf{a}-\mathbf{d})$, heptadecane $(\mathbf{e})$, tetradecane $(\mathbf{f})$, or eicosane $(\mathbf{g}, \mathbf{h})$ drops in emulsions, stabilized by $1.5 \mathrm{wt} \%$ of different surfactants: a, non-ionic Tween 60; b, c, non-ionic Brij 78; d, cationic CTAB; e, f, non-ionic Tween 40; $\mathbf{g}, \mathbf{h}$, Brij 78. Scale bars, $20 \mu \mathrm{m}$. 

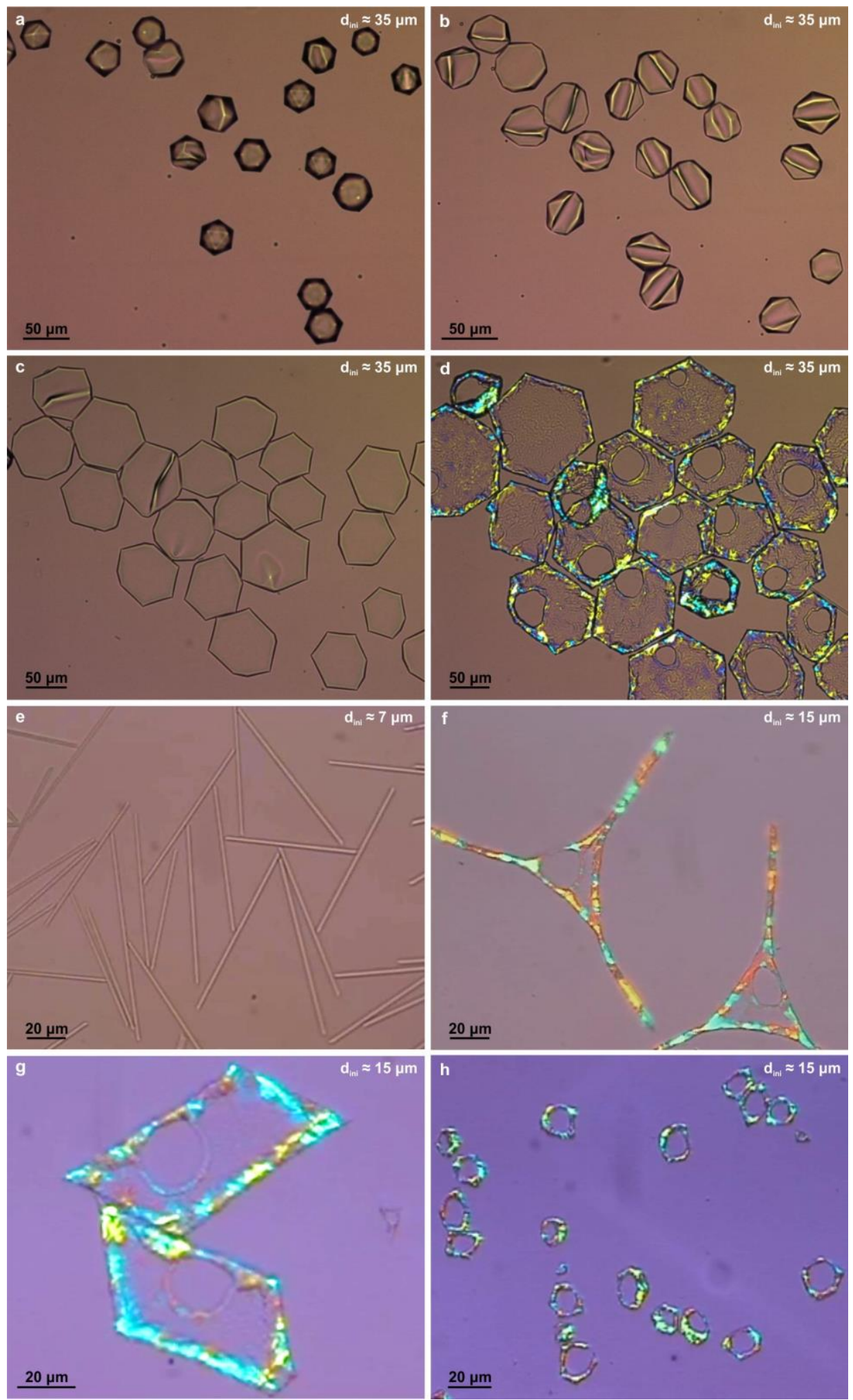

Extended Data Figure 2 Snapshot images of multiple hexadecane particles, obtained in 1.5 wt \% solutions of various surfactants. a-h, Tween 60 (a-e), Brij 58 (f) and Tween 40 (g, h). a-d, Consecutive images from the evolution of emulsion droplets stabilized by Tween 60. e, Rod-like particles before freezing. f, Frozen triangular particles. g, Frozen tetragonal platelets. h, Frozen toroidal particles. The initial drop sizes of the particles are indicated on the pictures. Cooling rates are $0.5 \mathrm{~K} \mathrm{~min}^{-1}$, except for $\mathbf{h}, 2 \mathrm{~K} \mathrm{~min}^{-1}$. 


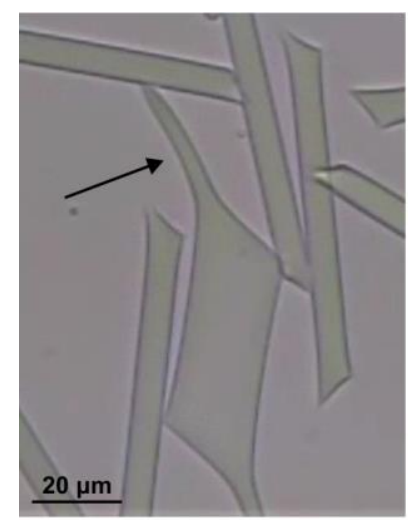

$t=0 s$

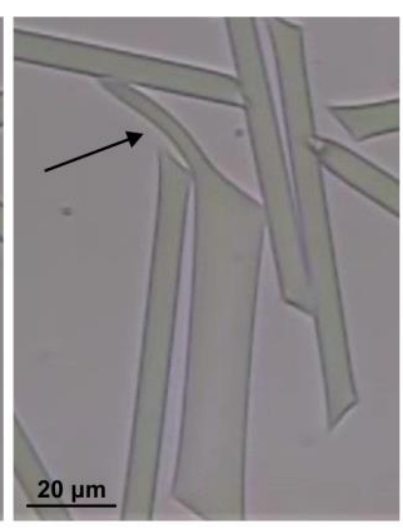

$t=6 s$

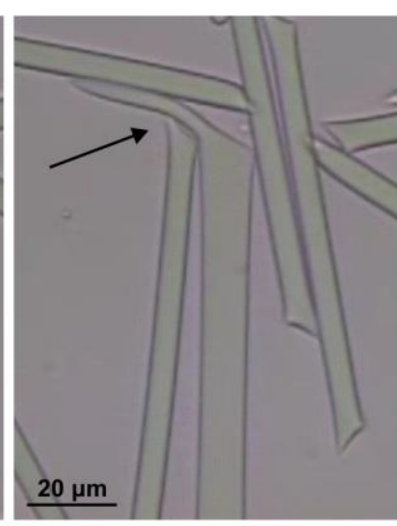

$t=10 s$

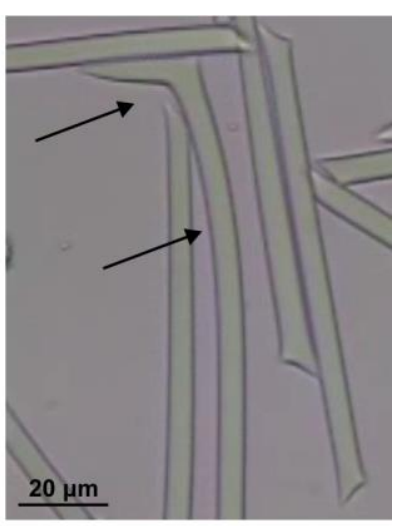

$t=15 s$

Extended Data Figure 3 Images proving that the deformed drops are still fluid. Extending drops collide with each other and bend, as shown with the black arrow. Images of hexadecane drops in $1.5 \mathrm{wt} \%$ aqueous solution of Brij 58, cooled with rate of $1 \mathrm{~K} \mathrm{~min}^{-1}$.
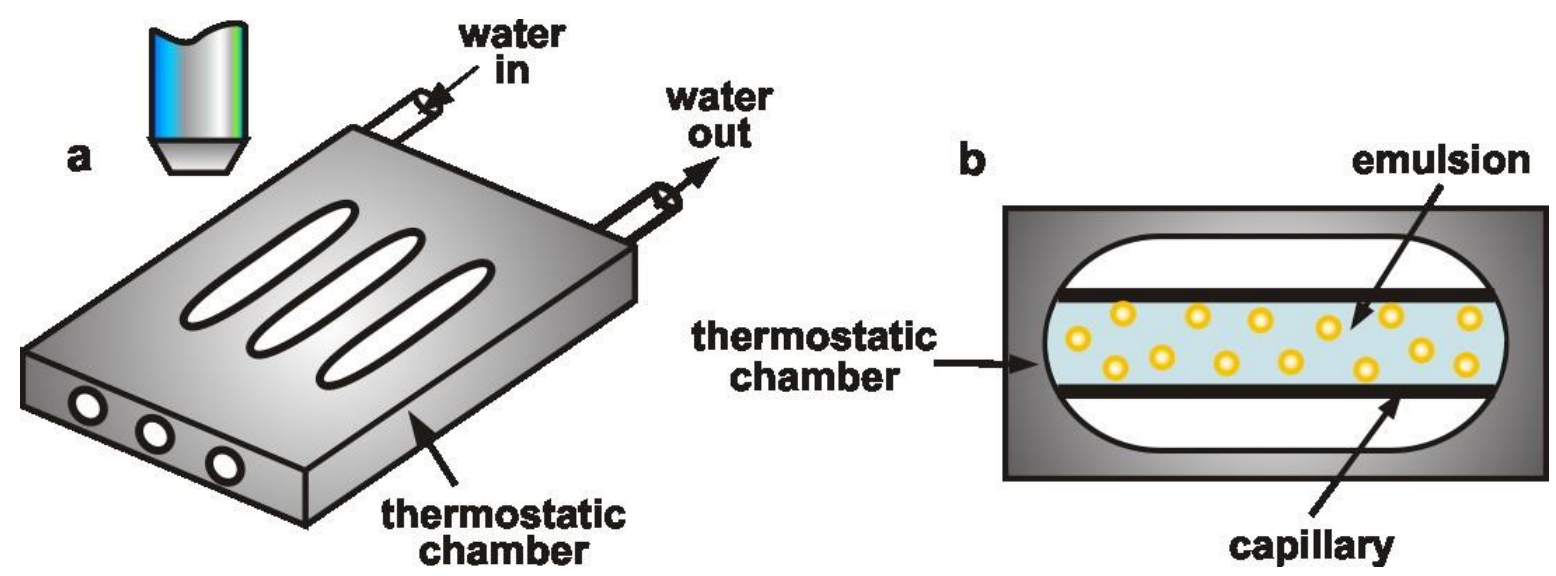

Extended Data Figure 4 Experimental setup. a, Schematic presentation of the cooling chamber with optical windows, used for microscope observation of the emulsion samples. $\mathbf{b}$, The studied emulsions are contained in glass capillaries, placed in the thermostatic chamber and observed through the optical windows.

Extended Data Table 1 Hydrophilic-lipophilic balance values of the used non-ionic surfactants

\begin{tabular}{|c|c|}
\hline Surfactant & HLB value \\
\hline Brij 58 & 15.7 \\
\hline Brij 78 & 15.3 \\
\hline Tween 40 & 15.5 \\
\hline Tween 60 & 14.9 \\
\hline
\end{tabular}

HLB, hydrophilic-lipophilic balance. 


\section{Appendix 1: Estimates of the energy balance upon drop deformation due to plastic layer formation:}

The formation of thin plastic phase at the drop surface and the concomitant drop deformation are related to changes in the drop free energy which might be estimated as follows:

The increase in the drop surface area, $\Delta A$, leads to an increase of the total drop surface energy $\Delta E_{S}=\gamma \Delta A$, where $\gamma$ is the interfacial tension. For simplicity here we assume that interfacial tensions of liquid oil-water and plastic phase-water interfaces are equal and neglect the interfacial tension between liquid oil and plastic phase. On the other hand, the formation of a thin plastic layer in the drop interior is a phase transition with an energy change of $\Delta E_{\mathrm{PL}}$ $=N_{\mathrm{PL}} \Delta \mu_{0}=\left(V_{\mathrm{PL}} / \mathrm{v}_{\mathrm{M}}\right) \Delta \mu_{0}$, where $N_{\mathrm{PL}}$ is the number of hydrocarbon molecules included in the plastic phase which, in turn, can be found as a ratio of the volume of the formed plastic layer, $V_{\mathrm{PL}}$, and the molecular volume of the hydrocarbon molecules, $\mathrm{v}_{\mathrm{M}}$. For undercooled drops the change in the standard chemical potential of the hydrocarbon molecules upon the spontaneous phase transition from isotropic liquid into plastic phase, $\Delta \mu_{0}=\mu_{\mathrm{PL}}-\mu_{\mathrm{OIL}}$, is a negative value. Therefore, the energy gain in the process of formation of the plastic phase is the one that compensates the expansion of the drop surface area and the related increase of the drop surface energy.

From the observed drop shapes we could estimate the molecular energy gain of plastic phase formation, $\Delta \mu_{0}$, in the systems studied. Upon flattening of a spherical drop with an initial radius $R$ into a circular platelet with a thickness around $2 r$, where $r$ is the characteristic radius of curvature of the platelet edges, one can estimate the radius of the formed platelet, $R_{\mathrm{P}}$, from the drop volume conservation. For simplicity in calculations, we present them for a diskshaped platelet with large aspect ratio, viz. with $r / R_{\mathrm{P}}<<1$. The estimates for other shapes of the deformed droplets are straightforward and lead to a different numerical pre-factor without changing the estimated order of magnitude and the conclusions.

From the drop volume conservation we estimate $R_{\mathrm{P}}$ as follows:

$$
(4 / 3) \pi R^{3} \approx 2 \pi r R_{\mathrm{P}}^{2} \text { hence } R_{\mathrm{P}} \approx\left(2 R^{3} / 3 r\right)^{1 / 2}
$$

Thus one can estimate the increase of the drop surface area upon platelet formation:

$$
\Delta A \approx 2 \pi R_{\mathrm{P}}^{2}+4 \pi r R_{\mathrm{P}}-4 \pi R^{2} \approx 4 \pi R^{2}(R / 3 r-1)
$$

where the assumption for a platelet with high aspect ratio, $r / R<<1$, was used.

As explained in the text and illustrated in Figure 4, plastic layers are formed only in the regions with high curvature. For the platelets these regions are located at the platelet perimeter only. Therefore, the volume of the formed plastic layer could be estimated as

$$
V_{\mathrm{PL}} \approx 2 \pi^{2} r R_{\mathrm{P}} h_{\mathrm{PL}}
$$


where $h_{\mathrm{PL}}$ is the thickness of the plastic layer formed. By comparing the surface energy loss with the plastic formation energy gain we can estimate the value of $\Delta \mu_{0}$ which is able to bring about the observed shape transformations:

$$
|\gamma \Delta A| \approx\left|\left(V_{\mathrm{PL}} / \mathrm{v}_{\mathrm{M}}\right) \Delta \mu_{0}\right|
$$

hence

$$
\left|\Delta \mu_{0}\right| \approx 2 \gamma R^{2}(R / 3 r-1) \mathrm{v}_{\mathrm{M}} /\left(\pi r R_{\mathrm{P}} h_{\mathrm{PL}}\right)
$$

Taking typical values of $R=5 \mu \mathrm{m}, r=1 \mu \mathrm{m}, R_{\mathrm{P}}=9 \mu \mathrm{m}, h_{\mathrm{PL}} \approx 0.3 \mu \mathrm{m}, \gamma=10^{-2} \mathrm{~J} / \mathrm{m}^{2}, \mathrm{v}_{\mathrm{M}} \approx$ $5 \times 10^{-28} \mathrm{~m}^{3}$ (for hexadecane), one estimates

$$
\Delta \mu_{0} \approx-2 \times 10^{-23} \mathrm{~J} \approx-0.005 k T
$$

where $k T$ is the thermal energy.

In a more formal and less physically transparent way, one can made the same estimate by considering the identity between the surface bending energy per unit area, introduced in the main text, $E_{\mathrm{B}} \approx K_{\mathrm{B}} / r^{2}$, and the corresponding plastic phase formation energy which (per unit area of the drop surface) is estimated as $\Delta \mu_{0} h_{\mathrm{PL}} / \mathrm{v}_{\mathrm{M}}$. Thus one estimates

$$
\Delta \mu_{0} \approx-K_{\mathrm{B}} \mathrm{V}_{\mathrm{M}} / r^{2} h_{\mathrm{PL}} \approx-2 \times 10^{-23} \mathrm{~J} \approx-0.005 k T
$$

where the bending energy was assumed to be $\approx 10^{-14} \mathrm{~J}$ for the reasons explained in the main text.

Note that $\Delta \mu_{0}$ has both enthalpic and entropic sub-components. The entropic component is negative upon phase transition which increases the molecular order, as for the liquid-rotator phase transition, studied in our experiments. Therefore, the entropic component opposes this phase transition. A negative enthalpic component, which is bigger in magnitude than the entropic component, is needed to counterbalance the entropic component and to induce such a phase transition. Each of the enthalpic and entropic components is of the order of $k T$ around the liquid-rotary phase transition, as measured by $\mathrm{DSC}^{1}$, whereas the estimated small value of $\approx-0.005 k T$ is the difference between these two components, needed to compensate for the increased surface area of the deforming droplets.

Let us emphasize that the above estimates are approximate and they aim only to demonstrate that the energy gain due to plastic phase formation is sufficient to explain the observed shape transformations. More complete analysis of the energy changes in the studied systems is postponed for a following, more detailed study.

1. Sirota, E. B., Singer, D. M., Phase transitions among the rotator phases of the normal alkanes, J. Chem. Phys. 101, 10873-10882 (1994). 